\title{
Loneliness and Emotional Support Predict Physical and Psychological Distress in Latinas with Breast Cancer and Their Supportive Partners
}

\author{
Chris Segrin ${ }^{1, *}$, Terry Badger ${ }^{2}$ and Alice Pasvogel ${ }^{2}$ \\ ${ }^{1}$ Department of Communication, University of Arizona, Tucson, AZ 85721, USA \\ ${ }^{2}$ College of Nursing, University of Arizona, Tucson, AZ 85721, USA
}

\begin{abstract}
Following predictions from the stress process model, associations between loneliness, emotional support, physical health, and psychological distress were tested in 115 Latinas with breast cancer and their supportive partners. Results showed that loneliness and emotional support were predictive of psychological distress and physical symptoms. Additionally, emotional support minimized the association between physical symptoms and psychological distress. There were strong individual-level effects for loneliness and emotional support on physical health and psychological distress, and evidence that a partner's loneliness also worsened psychological distress in the participants.
\end{abstract}

\section{INTRODUCTION}

Current estimates indicate that 1 in 3 Latinas will receive a diagnosis of cancer during her lifetime [1]. Although the incidence of breast cancer is lower in Latinas than in nonHispanic whites, there is growing evidence that the incidence is increasing with acculturation [2]. Latinas with breast cancer are also more likely to be diagnosed at later stages, with increased psychological distress and poorer physical health, than non-Hispanic Whites [3, 4]. Latino cancer patients report significantly more psychological distress, especially depression, and poorer social and health-related quality of life than non-Hispanic Whites [5, 6]. This is a significant issue in that psychological distress in cancer patients has been linked to decreased immune function [7], increased health care [8], and reduced long-term survival [9]. The experience of anxiety, for example, has been associated with increased symptom distress [10], adverse side-effects from chemotherapy [11], and early mortality [12] in breast cancer patients.

For many Latinas, breast cancer occurs in a larger context of challenging socio-economic and socio-political circumstances that only further compound the risk for psychological morbidity [13]. This poor psychological state, in turn, increases the risk for a range of negative health outcomes. Accordingly, the primary aim of this investigation is to examine the relationship between two key interpersonal relationship variables (i.e., loneliness and emotional support) as communication factors that might degrade or improve health-related quality of life and psychological quality of life in Latinas with breast cancer and their supportive partners.

*Address correspondence to this author at the Department of Communication, University of Arizona, Tucson, AZ USA, 85721;

E-mail: segrin@u.arizona.edu
The health communication and behavioral health literature have recently proliferated with studies documenting the pathogenic effects of loneliness on physical and mental health [14-16]. Loneliness occurs when there is a discrepancy between a person's desired and achieved level and quality of social contact [17]. Older adults who are lonely make more emergency hospital visits [18] and have a substantially higher risk of all-cause mortality, even after controlling for age, chronic medical conditions, functional impairments, and depression, compared to their nonlonely peers $[19,20]$. Loneliness appears to degrade health through mechanisms such as elevated systolic blood pressure [21], heightened stress responses [22, 23], high risk heart conditions [24], and poor sleep quality [25-27].

Social support is a communication behavior that plays a critical role in the maintenance or disruption of mental and physical health $[28,29]$. Socially supportive communication helps the recipient manage uncertainty and difficulties associated with the situation that she/he is in [30]. Social support involves the provision of various resources such as information, tangible assistance, or emotional comforting through interpersonal exchanges. Among the myriad forms of social support, health communication research has highlighted the value of and need for emotional support among breast cancer survivors [31, 32]. The relational message that is coincidental to socially supportive communication affirms the value of the recipient to the sender and clearly shows some level of care and concern for the recipient. The availability of such supportive communication has proven to be beneficial to the psychological well-being of Latinas with breast cancer [13] and for the population more generally, perceived social support is associated with lower health symptomatology [33, 34].

The family members and supportive partners of breast cancer survivors can also experience the psychological 
distress, disruption of daily routines, financial burdens, etc. that accompany cancer diagnosis and treatment [35]. Following diagnosis, partners often experience the same or higher levels of psychological distress as cancer survivors [36]. These findings are important because the psychological distress of supportive partners reduces their abilities to provide social support to survivors during illness, potentially worsening health outcomes $[37,38]$. Further, there is strong evidence that the emotional states of cancer survivors and their social network members are interdependent. The psychological and physical distress of breast cancer survivors and that of their supportive partners tends to fluctuate in concert [39-41].

The stress process model [42] was originally developed to explain and predict caregiver burden, but has since been fruitfully adapted to explain distress in both supportive partners and cancer survivors themselves [43]. The stress process model begins by noting the importance of the context of care. These are personal qualities such as age, being Latina, socioeconomic status, or family context that signifies where people stand within social strata that have unequal distributions of responsibilities and rewards. Context of care variables can minimize or exacerbate the risk for poor health outcomes. Many of the context of care variables for Latinas with breast cancer function to elevate risk for poor outcomes. The stress process model also explains that people have psychosocial resources as well as other stressors that directly influence or that may interact to influence psychological and physical health outcomes. People with better psychosocial resources are expected to have better health outcomes, whether they are the cancer survivor or that survivor's supportive partner. In contrast, those who are grappling with stressors in addition to cancer, or providing care and support to a cancer patient, are predicted to be at risk for experiencing a range of negative outcomes.

In the present investigation, we test some of the tenets of the stress process model in a sample of individuals who are otherwise at high risk for poor health outcomes by virtue of their exposure to precarious context of care issues (i.e., Latinas undergoing treatment for breast cancer and their supportive partners). Loneliness is treated as a significant stressor that increases the risk for poor health. Emotional support is conceptualized as a key psychosocial resource variable that could lessen the risk for poor health outcomes in this sample. For the purpose of testing these predictions, we measured two indicators of physical health (global ratings of health and physical symptom distress) and two indicators of psychological morbidity that are particularly common to cancer survivors and their supportive partners (depression and anxiety).

For many cancer patients the experience of physical symptoms (e.g., nausea, pain, dry mouth, sleep disturbance) can generate a state of psychological distress [44]. People understandably make negative inferences about their general health based on such symptoms and this can easily fuel psychological distress as the symptoms worsen. We predict that the association between physical well-being or distress and psychological distress will be moderated by loneliness (a stressor) and emotional support (a psychosocial resource). Specifically,

H1a: Loneliness will moderate the association between survivors' physical health symptoms and their psychological distress such that this relationship would be stronger as survivors' loneliness increases.

H1b: Emotional support will moderate the association between survivors' physical health symptoms and psychological distress such that this relationship will be stronger as survivors have less emotional support.

In recognition of a burgeoning literature showing interdependent quality of life in cancer survivors and their supportive partners, we attempt to extend the stress process model by testing loneliness (a stressor) and emotional support (a psychosocial resource) as predictors of physical and psychological health outcomes at the dyadic level. Specifically, it is predicted that:

H2: Loneliness will be negatively associated and emotional support will be positively associated with physical health at both the individual level (actor effect) and at the dyadic level (partner effect).

Tests of partner effects explore whether one dyad member's loneliness or available social support is predictive of the other dyad member's physical or psychological health. According to the stress process model, stressors (e.g., loneliness) should lessen people's abilities to provide care and assistance to their partners, whereas psychosocial resources (e.g., emotional support)should enhance their ability to provide care. It is through these processes that such dyadic effects on health outcomes are theoretically possible.

\section{METHOD}

\section{Participants}

Participants in this investigation were 115 dyads composed of a Latina with breast cancer (hereafter "survivors") and her supportive partner (hereafter "partners"). The survivors were on average 49.76 ( $S D=$ 10.94) years of age. They described their ethnic background as primarily "Mexican-American" (56\%), followed by "Hispanic/Latina" (37\%), with the remainder identifying as South or Central American. The majority $(60 \%)$ were married, had a high school education or less $(65 \%)$, and were disabled (38\%), unemployed (22\%), employed part time $(6 \%)$, or employed full time (14\%), with the remainder being retired or having some other employment status. Most (72\%) indicated that they had an annual household income of < $\$ 30,000$.

All of the survivors were within one year of a primary diagnosis of breast cancer and were in active treatment. Ninety-three of the survivors knew the stage of their breast cancer which was carcinoma in situ (2\%), stage I (18\%), stage II $(36 \%)$, stage III $(28 \%)$, or stage IV (16\%). For treatments, $69 \%$ had a partial or complete mastectomy, $79 \%$ were either currently receiving or had recently completed chemotherapy, $26 \%$ were either currently receiving or had recently completed radiation therapy, and $13 \%$ were currently receiving hormone blocking therapy. 
The partners who participated in this investigation were on average $43.30(S D=12.60)$ years of age with $29 \%$ males and $71 \%$ females. The relationship between the survivor and partner was spouse/significant other (24\%), sibling (21\%), mother (18\%), daughter (16\%), friend $(15 \%)$, with the remaining $6 \%$ being some other family relation (e.g., cousin, son). Forty-three percent of the partners resided with the survivor. Most of the partners also described themselves as either Hispanic/Latina/o (44\%) or Mexican-American (43\%). The partners were mostly married (67\%), although not necessarily to the survivor. Their highest level of education was high school or less $(50 \%)$, some college or college graduate $(45 \%)$, or post-graduate degree $(5 \%)$. The employment status of the partners was $49 \%$ full time, $17 \%$ unemployed, $14 \%$ part-time, $7 \%$ retired, $6 \%$ disabled, with the remainder having some other employment status. Their annual household income was $<\$ 30,000$ in $54 \%$ of the cases.

\section{Procedure}

Participants were part of a larger investigation of psychosocial interventions for breast cancer survivors and their supportive partners that was approved by an Institutional Review Board. All of the data presented in this report came from a baseline assessment taken before the commencement of the intervention. Participants were recruited from a local cancer center, oncologists' offices, support groups, and through self-referral after reading brochures displayed in various settings. Eligibility criteria for survivors included diagnosis of breast cancer, currently receiving adjuvant treatment for breast cancer, ability to speak either English or Spanish and talk on the telephone, no self-reported physical or cognitive disabilities that would prevent participation in the intervention, and availability of a partner who was willing to participate in the investigation. Survivors were asked to designate a supportive partner for participation in the study. This individual could be anyone whom they identified as a significant person in their cancer recovery and survivors were not restricted to selecting spouses or significant others. All of the baseline measures were taken over the telephone in either Spanish or English according to participants' preference by trained data collectors who recorded participants' responses on a computer as they were provided.

\section{Measures}

\section{Loneliness}

Participants completed the PROMIS [Patient-Reported Outcomes Measurement Information System] Social Isolation scale as a measure of their current level of loneliness [45]. The PROMIS instruments have been translated into Spanish and thoroughly tested for psychometric quality. This scale contains eight items that assess feelings indicative of loneliness and social isolation such as "I feel that people are around me but not with me," "I feel detached from other people," and "I feel isolated from others." Response options ranged from 1 (never) to 5 (always). Internal consistency reliability was $\alpha=.92$ for survivors and $\alpha=.94$ for partners.

\section{Emotional Support}

The PROMIS-Emotional Support scale [46] was administered to assess the extent to which participants felt that they had emotional support available when they needed it. This instrument asks respondents to indicate how often they feel that they have someone who provides different elements of emotional support. Sample items include, "I have someone to confide in or talk to about myself or my problems," "I have someone I trust to talk with about my feelings," and "I have someone with whom to share my most private worries and fears." Response options ranged from 1 (never) to 5 (always). Internal consistency reliability was $\alpha=$ .94 for survivors and $\alpha=.95$ for partners.

\section{Depression}

Symptoms of depression were assessed with the 20-item Center for Epidemiological Studies-Depression scale [47]. This instrument contains statements that reflect different symptoms of depression (e.g., "I felt depressed," "I felt that everything I did was an effort," and "I thought my life had been a failure"). Respondents are asked to indicate how frequently they have felt this way over the past 2 weeks with a scale that ranges from 0 (rarely or none of the time) to 3 (most or all of the time). Internal consistency reliability was $\alpha=.92$ for survivors and $\alpha=.91$ for partners.

\section{Anxiety}

Symptoms of Anxiety were assessed with the PROMISAnxiety scale [48]. This 8-item scale asks respondents to indicate how frequently they have experienced symptoms of anxiety such as fear, anxious misery, and hyperarousal. Sample items include "I felt anxious," "My worries overwhelmed me," and "I felt tense." Response options ranged from 1 (never) to 5 (always). Internal consistency reliability was $\alpha=.93$ for survivors and $\alpha=.89$ for partners.

\section{Global Health}

To measures participants' overall physical health, they were asked to complete the PROMIS-Global Health scale [49]. This 4-item measure contains statements about perceptions of general or global health (e.g., "In general, how would you rate your physical health," and "In general, would you say your health is") followed by response options that ranged from 1 (poor) to 5 (excellent). Internal consistency reliability was $\alpha=.87$ for survivors and $\alpha=.83$ for partners.

\section{Symptom Distress}

Distress associated with illness symptoms was assessed with the General Symptom Distress Scale (GSDS) [50]. The GSDS contains 12 symptoms that are commonly experienced by cancer patients but that are also experienced in conjunction with a wide variety of illnesses and ailments (e.g., nausea, cough, sleep difficulties). Respondents indicated whether they were experiencing each of the 12 symptoms at the present time, and these were summed to form a 0-12 scale, with higher scores indicating greater symptom distress. The internal consistency reliability was $\alpha$ $=.76$ for survivors and $\alpha=.77$ for partners. 


\section{RESULTS}

Hypothesis 1a predicted that loneliness would moderate the association between survivors' physical health symptoms and their psychological distress such that this relationship would be stronger as survivors' loneliness increased. This hypothesis was tested in a series of multiple regression analyses conducted in accord with recommendations put forth by Aiken and West [51], namely centering predictors, creating the interaction term as the product of the centered predictors, and post-hoc deconstruction of significant moderators with conditional effects of the IV on the DV calculated at $-1 \mathrm{SD}$, the mean, and $+1 \mathrm{SD}$ of the moderator. Because multiple regression is substantially underpowered to detect significant moderators in field research settings [52], we opted to deconstruct marginally significant effects ( $p=.06)$ for purposes of illustration, because of the otherwise conservative nature of these tests. For these analyses, global health and symptom distress were tested separately as independent variables predicting depression and anxiety, again in separate analyses. In all cases, loneliness was tested as the moderator variable. A correlation matrix of all the variables in these analyses appear in Table $\mathbf{1}$ and results of the multiple regression analyses appear in Table 2.

The results in Table $\mathbf{1}$ show that loneliness was significantly and positive associated with depression, anxiety, and symptom distress, and negatively associated with reports of global health. Results in Table $\mathbf{2}$ show that

Table 1. Correlations among major study variables for survivors.

\begin{tabular}{|c|c|c|c|c|c|c|}
\hline & 1 & 2 & 3 & 4 & 5 & 6 \\
\hline 1. Global Health & -- & & & & & \\
\hline 2. Symptom Distress & $.42 * * *$ & -- & & & & \\
\hline 3. Loneliness & $-.28 * *$ & $.31^{* *}$ & -- & & & \\
\hline 4. Emotional Support & $.31 * *$ & -.17 & $-.39 * * *$ & -- & & \\
\hline 5. Depression & $-.52 * * *$ & $.58 * * *$ & $.52 * * *$ & $-.39 * * *$ & -- & \\
\hline 6. Anxiety & $-.39 * * *$ & $.53 * * *$ & $.48 * * *$ & $-.42 * * *$ & $.80^{* * *}$ & -- \\
\hline
\end{tabular}

Note. $* * p<.01 . * * * p<.001$.

Table 2. Loneliness and emotional support as moderators of the association between physical health and psychological distress.

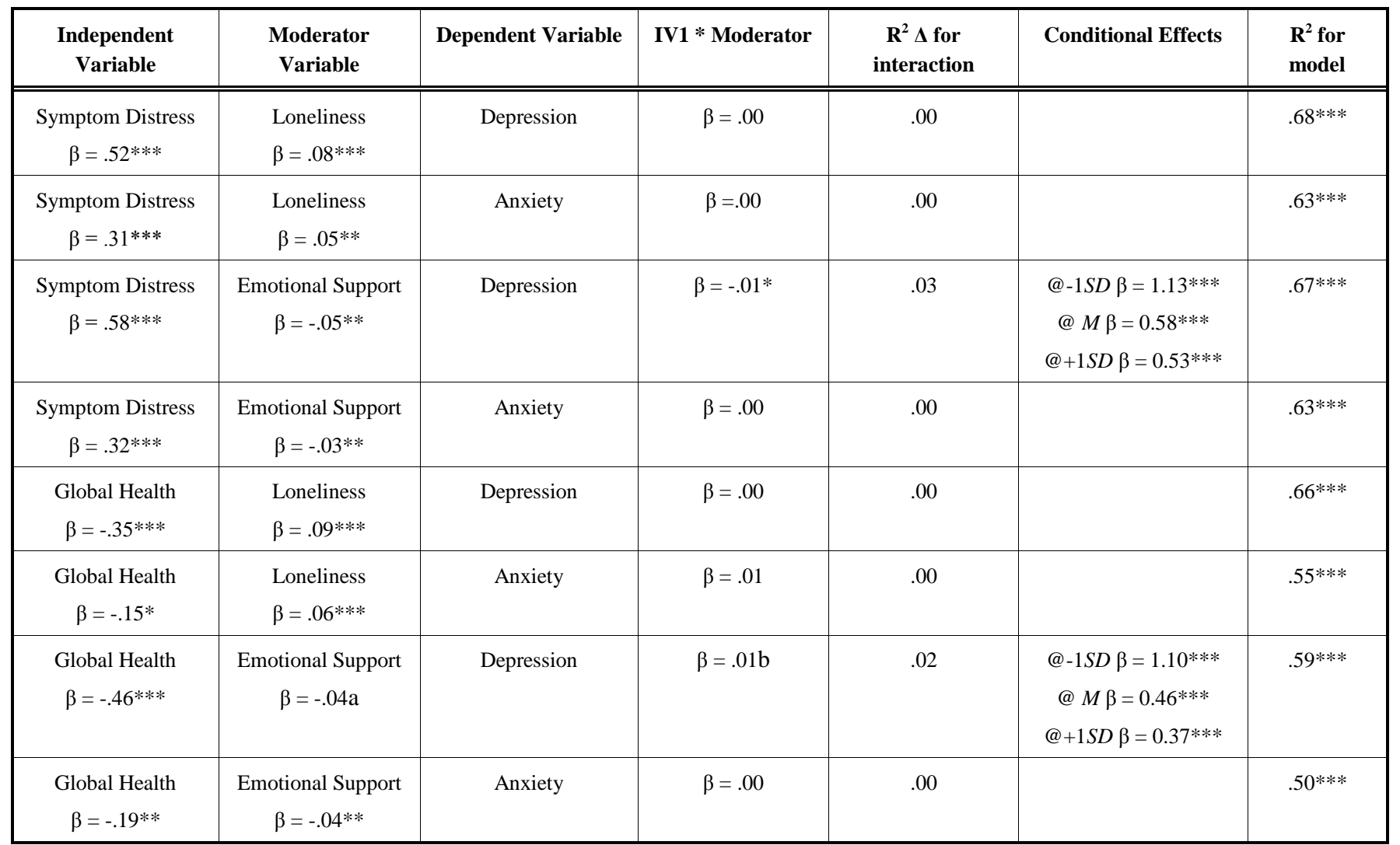

Note. $* p<.05 . * * p<.01 . * * * p<.001{ }_{\text {a }} \mathrm{p}=.07$. b $\mathrm{p}=.06$. 
loneliness was not a significant moderator of any of the associations between physical and psychological well-being, disconfirming H1a. However, the significant main effects indicate that in all cases loneliness was a significant predictor of depression and anxiety, even when controlling for symptom distress or global health.

Hypothesis H1b predicted that emotional support would moderate the association between survivors' physical health symptoms and psychological distress such that this relationship would be stronger as survivors had less emotional support. This hypothesis was tested in the same manner as H1a. These results also appear in Table $\mathbf{2}$.

Results in Table $\mathbf{2}$ indicate that emotional support was a significant moderator of the association between symptom distress and depression and there was a marginal moderation of the global health-depression association. In both cases, the pattern of conditional effects was as predicted, namely that the association between poor physical health and depression becomes stronger as levels of emotional support available to the survivor decrease (see Table 2 for conditional effects). Results of these regression analyses did not indicate any significant moderation of the symptom distress-anxiety association or the global health-anxiety association by emotional support. The significant main effects show that emotional support was predictive of lower depression and anxiety, even when controlling for symptom distress or global health. Consequently, these results provide partial support for the hypothesized moderation of the physical health-psychological distress association by emotional support.

Hypothesis 2 predicted that loneliness and emotional support would each be predictive of physical health and psychological distress at both the individual and dyadic level. Tests of dyadic interdependence were conducted with structural equation modeling in AMOS 20.0 using maximum likelihood estimation, following the actor-partner interdependence model (APIM) [53, 54]. In these analyses, actor effects (i.e., survivors' IV $\rightarrow$ survivors' DV, partners' IV $\rightarrow$ partners' DV) and partner effects (i.e., survivors' IV $\rightarrow$ partners' DV, partners' IV $\rightarrow$ survivors' DV) are simultaneously estimated, each controlling for the other. These estimates also control for the dyad's initial similarity on the IV, as estimated by the intraclass correlation coefficient (ICC). Error terms for the survivors' and partners' DVs are allowed to be correlated. For each indicator of psychological distress and each indicator of physical health, two APIMs were analyzed, one with loneliness as the IV and one with emotional support as the IV. Accordingly, there were 8 APIMs estimated overall. In each analysis, two models were compared: one in which all of the paths from survivor variables were constrained equal to their respective paths from the partner variables, and one in which all paths were unconstrained and thus free to vary. Comparison of the fit of these two models with the chisquare difference test consistently indicated that the constrained model had the best fit and thus all subsequent results are from the more parsimonious constrained models. Results of these analyses appear in Table 2.

The results in Table $\mathbf{2}$ reveal strong and ubiquitous actor effects for loneliness as a predictor of both psychological distress and physical health. For both survivors and partners, high levels of loneliness were predictive of high levels of depression, anxiety, and symptom distress, and lower rating of global health (see Table 2 for standardized regression coefficients). At the individual level, loneliness had negative associations with multiple indicators of quality of life. At the dyadic level, there was evidence of two statistically significant partner effects from one person's loneliness to the other's anxiety. For both the survivor $\rightarrow$ partner path and the partner $\rightarrow$ survivor path these effects were significant indicating that loneliness is not only predictive of one's own higher anxiety but higher anxiety in one's partner as well. There were also two marginal partner effects $(p=.09)$ for loneliness predicting depression. These findings suggest that one's own loneliness is associated with somewhat higher levels of depression in the dyadic partner.

Results for emotional support, that appear on the bottom half of Table 2, also show strong and ubiquitous actor effects. Low levels of emotional support predicted higher levels of depression, anxiety, and symptom distress, and low ratings of global health for both survivors and partners. There were, however, no statistically significant partner effects associated with emotional support. The effects of emotional support on well-being therefore appear to be confined to the individual rather than dyadic level.

\section{DISCUSSION}

This study was designed to test the potentially deleterious effects of loneliness and the salutary effects of emotional support on physical and psychological distress among Latinas with breast cancer and their supportive partners. The demographic context of care variables clearly illustrate how this population is already at risk for poor outcomes by virtue of low income, high unemployment, modest education, and for the Latina cancer survivors, advanced disease stage in many cases. The results showed that loneliness and emotional support were significantly associated with physical and psychological health outcomes in breast cancer survivors. The availability of emotional support minimized the association between physical health symptoms and depression in Latinas with breast cancer. For both cancer survivors and their supportive partners, loneliness appeared detrimental to, and emotional support beneficial to, psychological and physical health. Although these relationships were evident largely at the individual level, findings from this study revealed some evidence of dyadic partner effects whereby one person's loneliness was also associated with increased depression and anxiety in his or her partner. These results illustrate how quality of life while undergoing cancer treatment is potentially degraded by loneliness experienced by the self and one's partner.

According to the stress process model $[42,43]$, stressors that are incidental to cancer diagnosis and treatment will compromise health outcomes. There is substantial evidence documenting loneliness as a subjectively stressful experience $[27,55]$. Consistent with that model, the results of this study showed that loneliness is associated with greater depression and anxiety, even when controlling for global health and symptom distress. This finding is understandable to the extent that loneliness may be a proxy for lack of supportive 
relationships among the cancer survivors who participated in this study. Social support is an effective buffer against the ill effects of stress [56, 57]. People who experience loneliness are likely lacking in this important resource that could otherwise help them appraise and cope with potentially distressing physical symptoms. To this point, there was complementary evidence that emotional support was also predictive of better physical and psychological health outcomes and that it also moderated the association between physical health and depression. Specifically, the poor healthdepression association was weakest among those cancer survivors who reported the highest levels of emotional support available.

Results from actor-partner interdependence analysis Table 3 revealed ubiquitous actor effects for the association between loneliness and psychological distress and between low emotional support and psychological distress. These findings are consistent with the stress process model [42] which posits that stressors (e.g., loneliness) and psychosocial resources (e.g., emotional support) that are incidental to major health problems will complicate or potentially improve clinical outcomes. These findings provide further support for theoretical models of loneliness as a hazard to health [14] while adding to the growing health communication literature that underscores the importance of emotional support to breast cancer survivors [58, 59], especially Latinas [60]. It is particularly noteworthy that all participants-survivors or partners-who reported high levels of loneliness reported poor health relative to those with low levels of loneliness. Additionally, survivors and partners with available emotional support reported better health than those without access to this psychosocial resource.

Although the partner effects were generally weak in magnitude and statistically nonsignificant, there was evidence of partner effects for the association between loneliness and psychological distress (i.e., anxiety and depression). There are several potential explanations for these effects. First, they could be an artifact due to the tendency of loneliness to cluster in families and other social networks $[61,62]$. If lonely people experience psychological distress, it may seem possible that the loneliness of their social network members predicts their own psychological distress by virtue of this agglomeration effect. However, the APIM analyses conducted in this study estimate partner effects while controlling for actor effects. In other words, the partner's loneliness predicts the actor's psychological distress, above and beyond that which is already predicted by the actor's own level of loneliness. This suggests several alternative possibilities. One is that the stress of loneliness may hinder the participant's wherewithal to provide support and companionship to his or her partner, resulting in a partner who experiences increased psychological distress. Second, the stress of loneliness in a partner may spill over to the self. In other words, a partner's loneliness might add a burden to the self, resulting in higher psychological distress. At this time these explanations are speculative but deserving of further research and investigation.

There are several features of this investigation that naturally produce scope conditions around interpretations of these findings. First, the cross-sectional nature of the study does not allow for unambiguous estimation of causal ordering. Because the variables measured in this investigation are not amenable to experimental manipulation, longitudinal research would be the best alternative for more accurately ordering these variables. Noting that, it remains unlikely that a clear cause-effect model will emerge in the study of loneliness and health. Poor health could easily generate feelings of loneliness as people take increased time away from work, friends, and family because of their illness. Just as easily, loneliness could exert a negative effect on health through processes such as compromised health behaviors, heightened stress reactions, sleep disruption, and diminished access to social support [14]. Second, to qualify for inclusion in this investigation, cancer survivors had to secure the participation of a supportive partner who would also participate. Accordingly, those living in the most socially austere conditions were likely filtered out, resulting in an artifactually restricted range of loneliness scores. This makes the statistically significant findings from this study all

Table 3. Results of actor-partner interdependence analyses of loneliness and emotional support as predictors of psychological distress and physical health actor effects partner effects model fit.

\begin{tabular}{|c|c|c|c|c|c|c|c|c|c|}
\hline $\begin{array}{c}\text { Independent } \\
\text { Variable }\end{array}$ & $\begin{array}{l}\text { Dependent } \\
\text { Variable }\end{array}$ & ICC & $\beta_{\mathrm{s}_{\rightarrow} \mathrm{s}}$ & $\boldsymbol{\beta}_{\mathbf{P}_{\rightarrow}} \mathbf{P}$ & $\beta_{\mathrm{S}_{\rightarrow}} \mathrm{P}$ & $\boldsymbol{\beta}_{\mathbf{P}_{\rightarrow} S}$ & $\chi^{2} / \mathbf{d f}$ & CFI & RMSEA \\
\hline Loneliness & Anxiety & -.05 & $.47 * * *$ & $.57 * * *$ & $.15^{* *}$ & $.18 * *$ & 1.59 & 0.98 & .07 \\
\hline Loneliness & Depression & -.05 & $.53 * * *$ & $.64 * * *$ & $.08_{\mathrm{a}}$ & $.10_{\mathrm{a}}$ & 1.02 & 1.00 & .00 \\
\hline Loneliness & Global Health & -.05 & $-.29 * * *$ & $-.35 * * *$ & -.03 & -.04 & 0.03 & 1.00 & .00 \\
\hline Emotional Support & Anxiety & .02 & $-.39 * * *$ & $-.46^{* * *}$ & .01 & -.01 & 0.52 & 1.00 & .00 \\
\hline Emotional Support & Depression & .02 & $-.39 * * *$ & $-.45 * * *$ & -.02 & -.02 & 0.09 & 1.00 & .00 \\
\hline Emotional Support & Global Health & .02 & $.30 * * *$ & $.36 * * *$ & .06 & .07 & 0.28 & 1.00 & .00 \\
\hline Emotional Support & Symptom Distress & .02 & $-.33^{* * *}$ & $-.46 * * *$ & .00 & -.01 & 2.13 & 0.93 & .10 \\
\hline
\end{tabular}

Note: ICC $=$ intraclass correlation [or the proportion of the variance in the dependent variable accounted for by couple membership in each model], $\mathrm{CFI}=$ comparative fit index, RMSEA = root mean square error of approximate. $\mathrm{S}=$ Survivor. $\mathrm{P}=$ Partner. $* * p<.01, * * * p<.001$. a $p=.09$. 
the more remarkable.

In conclusion, this investigation highlights the importance of emotional support and loneliness for Latina cancer survivors and their supportive partners who are often coping with this major threat to well-being under what are already stressful circumstances. Loneliness was consistently associated with indicators of poor health and psychological distress. In contrast, emotional support was associated with better physical and mental health profiles in both survivors and partners. This suggests that those working in a clinical context may benefit from asking some simple questions about loneliness and available social support when providing services to cancer survivors and their supportive partners. There is reason to believe that these two variables may be risk and resiliency factors in the prediction of poor physical and mental health outcomes. Particularly in the case of emotional support, the resiliency factor is amenable to interventions that could be part of a comprehensive cancer treatment plan. Furthermore, there is at least a theoretical rational for expecting such interventions to coincidentally lessen loneliness, further meeting the needs of a population at high risk for poor health outcomes when confronted with a major illness such as breast cancer.

\section{CONFLICT OF INTEREST}

The authors confirm that this article content has no conflict of interest.

\section{ACKNOWLEDGEMENTS}

Author Note: This study was funded by a grant from the American Cancer Society, Terry Badger and Chris Segrin co-PIs.

\section{REFERENCES}

[1] American Cancer Society. Cancer Facts \& Figures, 2011. Atlanta, GA: American Cancer Society 2011.

[2] Kagawa-Singer M, Dadia AV, Yu MC, Surbone A. Cancer, culture and health disparities: Time to chart a new course. CA Cancer J Clin 2010; 60(1): 12-39.

[3] Buki LP, Garces DM, Hinestrosa MC, Kogan L, Carrillo IY, French B. Latina breast cancer survivors' lived experiences: diagnosis, treatment, and beyond. Cultural Diversity \& Ethnic Minority Psychology 2008; 14(2): 163-7.

[4] Napoles-Springer AM, Ortiz C, O'Biren H, Diaz-Mendez M, PerezStable EJ. Use of cancer support groups among Latina breast cancer survivors. J Cancer Survivorship 2007; 1: 193-205.

[5] Aguado Loi CX, Baldwin JA, McDermott RJ, et al. Risk factors associated with increased depressive symptoms among Latinas diagnosed with breast cancer within 5 years of survivorship. Psycho-oncology 2013; 22(12): 2779-88.

[6] Luckett T, Goldstein D, Butow PN, et al. Psychological morbidity and quality of life of ethnic minority patients with cancer: a systematic review and meta-analysis. Lancet Oncol 2011; 12(13): 1240-8.

[7] Giese-Davis J, Wilhelm FH, Conrad A, et al. Depression and stress reactivity in metastatic breast cancer. Psychosom Med 2006; 68(5): 675-83.

[8] Bambauer KZ, Zhang B, Maciejewski PK, et al. Mutuality and specificity of mental disorders in advanced cancer patients and caregivers. Soc Psychiatry Psychiatr Epidemiol 2006; 41(10): 81924.

[9] Giese-Davis J, Collie K, Rancourt KM, Neri E, Kraemer HC, Spiegel D. Decrease in depression symptoms is associated with longer survival in patients with metastatic breast cancer: a secondary analysis. J Clin Oncol 2011; 29(4): 413-20.
[10] Badger TA, Segrin C, Dorros SM, Meek P, Lopez AM. Depression and anxiety in women with breast cancer and their partners. Nurs Res 2007; 56: 44-53.

[11] Cameron LD, Leventhal H, Love RR. Trait anxiety, symptom perceptions, and illness-related responses among women with breast cancer in remission during a tamoxifen clinical trial. Health Psychol 1998; 17: 459-69.

[12] Groenvold M, Petersen MA, Idler E, Bjorner JB, Fayers PM, Mouridsen HT. Psychological distress and fatigue predicted recurrence and survival in primary breast cancer patients. Breast Cancer Res Treat 2007; 105(2): 209-19.

[13] Galvan N, Buki LP, Garces DM. Suddenly, a carriage appears: social support needs of Latina breast cancer survivors. J Psychosocial Oncol 2009; 27(3): 361-82.

[14] Cacioppo JT, Hawkley LC, Crawford LE, et al. Loneliness and health: potential mechanisms. Psychosom Med 2002; 64(3): 40717.

[15] Segrin C, Domschke T. Social support, loneliness, recuperative processes, and their direct and indirect effects on health. Health Commun 2011; 26(3): 221-32.

[16] Shankar A, McMunn A, Banks J, Steptoe A. Loneliness, social isolation, and behavioral and biological health indicators in older adults. Health Psychol 2011; 30(4): 377-85.

[17] Peplau LA, Perlman D. Perspectives on loneliness. In: Perlman LAPD, editor. Loneliness: A sourcebook of current theory, research and therapy. New York: Wiley 1982; p. 1-18.

[18] Molloy GJ, McGee HM, O'Neill D, Conroy RM. Loneliness and emergency and planned hospitalizations in a community sample of older adults. J Am Geriatr Soc 2010; 58(8): 1538-41.

[19] Patterson AC, Veenstra G. Loneliness and risk of mortality: a longitudinal investigation in Alameda County, California. Soc Sci Med 2010; 71(1): 181-6.

[20] Shiovitz-Ezra S, Ayalon L. Situational versus chronic loneliness as risk factors for all-cause mortality. Int Psychogeriatr 2010; 22(3): 455-62.

[21] Hawkley LC, Masi CM, Berry JD, Cacioppo JT. Loneliness is a unique predictor of age-related differences in systolic blood pressure. Psychol Aging 2006; 21(1): 152-64.

[22] Burke TJ, \& Segrin, C. Bonded of struck: Effects of personal and constraint commitment on loneliness and stress. Pers Indiv Diff 2014; 64: 101-6.

[23] Steptoe A, Owen N, Kunz-Ebrecht SR, Brydon L. Loneliness and neuroendocrine, cardiovascular, and inflammatory stress responses in middle-aged men and women. Psychoneuroendocrinology 2004; 29(5): 593-611.

[24] Sorkin D, Rook KS, Lu JL. Loneliness, lack of emotional support, lack of companionship, and the likelihood of having a heart condition in an elderly sample. Ann Behav Med 2002; 24(4): 2908.

[25] Cacioppo JT, Hawkley LC, Berntson GG, et al. Do lonely days invade the nights? Potential social modulation of sleep efficiency. Psychol Sci 2002; 13(4): 384-7.

[26] Segrin C, Burke TJ. Loneliness and Sleep Quality: Dyadic Effects and Stress Effects. Behav Sleep Med 2014.

[27] Segrin C, Passalacqua SA. Functions of loneliness, social support, health behaviors, and stress in association with poor health. Health Commun 2010; 25(4): 312-22.

[28] House JS, Landis KR, Umberson D. Social relationships and health. Science 1988; 241(4865): 540-5.

[29] House JS, Umberson D, Landi KR. Structures and processes of social support. Ann Rev Soc 1988; 14: 293-318.

[30] Albrecht TL, Goldsmith DJ. Social support, social networks, and health. In: Thompson TL, Dorsey AM, Miller KI, Parrott R, Eds. Handbook of health communication. Mahwah, NJ: Lawrence Erlbaum Associates 2003; pp. 263-84.

[31] Kim E, Han JY, Moon TJ, Shaw B, Shah DV, McTavish FM, et al. The process and effect of supportive message expression and reception in online breast cancer support groups. Psycho-Oncology 2012; 21(5): 531-40

[32] Lee SY, Hawkins R. Why do patients seek an alternative channel? The effects of unmet needs on patients' health-related Internet use. J Health Commun 2010; 15(2): 152-66.

[33] Lett HS, Blumenthal JA, Babyak MA, et al. Social support and prognosis in patients at increased psychosocial risk recovering from myocardial infarction. Health Psychol 2007; 26(4): 418-27. 
[34] Wills TA, Shinar O. Measuring perceived and received social support. In: Cohen LGU, Gottlieb BH, Eds. Social support measurement and intervention Oxford, UK: Oxford University Press 2000; pp. 86-133.

[35] Westman M, Keinan G, Roziner I, Benyamini Y. The crossover of perceived health between spouses. J Occup Health Psychol 2008; 13(2): 168-80.

[36] Rabin EG, Heldt E, Hirakata VN, Bittelbrunn AC, Chachamovich E, Fleck MP. Depression and perceptions of quality of life of breast cancer survivors and their male partners. Oncol Nurs Forum 2009; 36(3): E153-8.

[37] Ell K, Nishimoto R, Mediansky L, Mantell J, Hamovitch M. Social relations, social support and survival among patients with cancer. J Psychosom Res 1992; 36(6): 531-41.

[38] Given B, Given CW, Sikorskii A, Jeon S, Sherwood P, Rahbar M. The impact of providing symptom management assistance on caregiver reaction: results of a randomized trial. J Pain Symptom Manage 2006; 32(5): 433-43.

[39] Northouse L, Templin T, Mood D. Couples' adjustment to breast disease during the first year following diagnosis. J Behav Med 2001; 24(2): 115-36.

[40] Segrin C, Badger T. Interdependent psychological distress between Latinas with breast cancer and their supportive partners. J Latina/o Psychol 2013; 1: 21-34.

[41] Segrin C, Badger T, Dorros SM, Meek P, Lopez AM. Interdependent anxiety and psychological distress in women with breast cancer and their partners. Psycho-oncology 2007; 16(7): 634-43.

[42] Pearlin L, Mullan JT, Semple SJ, Skaff MM. Caregiving and the stress process: An overview of concepts and their measures. Gerontologist 1990; 30: 583-94.

[43] Gaugler JE, Linder J, Given CW, Kataria R, Tucker G, Regine WF. Family cancer caregiving and negative outcomes: the direct and mediational effects of psychosocial resources. J Fam Nurs 2009; 15(4): 417-44

[44] Portenoy RK, Thaler HT, Kornblith AB, et al. Symptom prevalence, characteristics and distress in a cancer population. Qual Life Res 1994; 3(3): 183-9.

[45] Hahn EA, DeWalt DA, Bode RK, Garcia SF, DeVellis RF, Correia $\mathrm{H}$, et al. New English and Spanish social health measures will facilitate evaluating health determinants. Health Psychol 2014; 33(5): 490-9.

[46] Hahn EA, Devellis RF, Bode RK, Garcia SF, Castel LD, Eisen SV, et al. Measuring social health in the patient-reported outcomes measurement information system [PROMIS]: item bank development and testing. Qual Life Res 2010; 19(7): 1035-44.
[47] Radloff LS. The CES-D Scale: A self-report depression scale for research in the general population. Appl Psychol Measur 1977; 1(3): 385-401.

[48] Pilkonis PA, Choi SW, Reise SP, et al. Item banks for measuring emotional distress from the Patient-Reported Outcomes Measurement Information System (PROMIS(R)): depression, anxiety, and anger. Assessment 2011; 18(3): 263-83.

[49] Hays RD, Bjorner JB, Revicki DA, Spritzer KL, Cella D. Development of physical and mental health summary scores from the patient-reported outcomes measurement information system (PROMIS) global items. Qual Life Res 2009; 18(7): 873-80.

[50] Badger TA, Segrin C, Meek P. Development and validation of an instrument for rapidly assessing symptoms: the general symptom distress scale. J Pain Symptom Manage 2011; 41(3): 535-48.

[51] Aiken LS, West SG. Multiple regression: testing and interpreting interactions. Thousand Oaks, CA: Sage 1991.

[52] McClelland GH, Judd CM. Statistical difficulties of detecting interactions and moderator effects. Psychol Bull 1993; 114(2): 37690.

[53] Kenny DA, Cook, WL. Partner effects in relationship research conceptual issues, analytic difficulties, and illustrations. Pers Relat 1999; 6: 433-88.

[54] Kenny DA, Kashy, DA, Cook WL, Eds. Dyadic data analysis. New York: Guilford Press 2006.

[55] Hawkley LC, Burleson MH, Berntson GG, Cacioppo JT. Loneliness in everyday life: cardiovascular activity, psychosocial context, and health behaviors. J Pers Soc Pychol 2003; 85(1): 10520 .

[56] Cohen S, Wills TA. Stress, social support, and the buffering hypothesis. Psychol Bull 1985; 98(2): 310-57.

[57] Uchino BN. Understanding the links between social support and physical health. Persp Psychol Sci 2009; 4: 236-55.

[58] Fisher C. Coping with breast cancer across adulthood: emotioanl support communication in the mother-daughter bond. J Appl Commun Res 2010; 27: 361-82.

[59] Leung J, Pachana NA, McLaughlin D. Social support and healthrelated quality of life in women with breast cancer: a longitudinal study. Psycho-oncology 2014; 23(9): 1014-20.

[60] Lopez-Class M, Perret-Gentil M, Kreling B, Caicedo L, Mandelblatt J, Graves KD. Quality of life among immigrant Latina breast cancer survivors: realities of culture and enhancing cancer care. J Cancer Educ 2011; 26(4): 724-33.

[61] Cacioppo JT, Fowler JH, Christakis NA. Alone in the crowd: the structure and spread of loneliness in a large social network. J Pers Soc Pychol 2009; (6): 977-91.

[62] Segrin C, Burke TJ, Dunivan, M. Loneliness and poor health within families. J Soc Pers Relat 2012; 29: 597-611.

This is an open access article licensed under the terms of the Creative Commons Attribution Non-Commercial License (http://creativecommons.org/licenses/by-nc/3.0/) which permits unrestricted, non-commercial use, distribution and reproduction in any medium, provided the work is properly cited. 